\title{
Intelligent Support System for Healthcare Logistics 4.0 Optimization in the Covid Pandemic Context
}

\author{
Paul-Eric Dossou ${ }^{1,2 *}$, Luiza Foreste ${ }^{1}$, Eric Misumi ${ }^{2}$ \\ ${ }^{1}$ Department of Technological, Societal Transition, ICAM Paris-Sénart, Lieusaint, France \\ ${ }^{2}$ Splott, AME, University of Gustave Eiffel, Marne-La-Vallée, France \\ Email: *paul-eric.dossou@icam.fr
}

How to cite this paper: Dossou, P.-E., Foreste, L. and Misumi, E. (2021) Intelligent Support System for Healthcare Logistics 4.0 Optimization in the Covid Pandemic Context. Journal of Software Engineering and Applications, 14, 233-256. https://doi.org/10.4236/jsea.2021.146014

Received: April 25, 2021

Accepted: June 18, 2021

Published: June 21, 2021

Copyright $\odot 2021$ by author(s) and Scientific Research Publishing Inc. This work is licensed under the Creative Commons Attribution International License (CC BY 4.0).

http://creativecommons.org/licenses/by/4.0/

\begin{abstract}
The covid pandemic points out inconsistencies and points to improve in the organization of healthcare logistics. Indeed, the dangerousness and the propagation process of the virus imply to increase health security (patient and personal health). In this context, healthcare logistics flows require a new and safety organization improving the hospital performance. The purpose of this paper consists in optimizing healthcare logistics flows by solving problems associated to the internal logistics such as reduction of the personal health wasting time and the protection of both patients and personal health. Then, the methodology corresponds to the use of the hospital sustainable digital transformation as a response to healthcare flows and safety problems. Indeed, social, societal and environmental aspects have to be considered in addition to new technologies such as artificial intelligence (AI), Internet of Things (IoTs), Big data and analytics. These parameters could be used in the healthcare for increasing doctor, nurse, caregiver performance during their daily operations, and patient satisfaction. Indeed, this hospital digital transformation requires the use of large data associated to patients and personal health, algorithms, a performance measurement tool (actual and future state) and a general approach for transforming digitally the hospital flows. The paper findings show that the healthcare logistics performance could be improved with a sustainable digital transformation methodology and an intelligent software tool. This paper aims to develop this healthcare logistics 4.0 methodology and to elaborate the intelligent support system. After an introduction presenting the common hospital flows and their main problems, a literature review will be detailed for showing how existing concepts could contribute to the elaboration of a structured methodology. The structure of the intelligent software tool for the healthcare digital transformation and the tool develop-
\end{abstract}


ment processes will be presented. An example will be given for illustrating the development of the tool.

\section{Keywords}

Healthcare Logistics 4.0, Industry 4.0, Lean Manufacturing, Artificial Intelligence, Intelligent Support System, IoT, Big Data Analytics

\section{Introduction}

Many countries in the world are confronted with problems related to covid pandemic management in hospitals. For instance, in France problems were detected on how to manage processes of masks, safety clothes, respirators, patient beds in intensive care, and both patient and personal health security. Indeed, the vaccine supply chain management has decreased the healthcare logistics performance. The problems that have been accentuated during this crisis are numerous but could be summarized around how to improve the hospital flows performance and to consider both personal health and patient security. Many tools and methodologies have been used for managing the actual hospital organization, but as the results in European countries have shown during the first wave, their success was moderated. In this context, finding solutions to increase the level of logistics performance and ensuring patient and personal health security is required.

Despite the great importance of Industry 4.0 main concepts and technologies in companies, they are not yet sufficiently integrated into hospitals. Nowadays, AGVs are already used for transporting goods inside the hospital and cobots/ robots are employed in the doctor's routine helping them in daily tasks. The existing digital transformation methods are mainly based on the use of new technologies and tools and correspond to reasons that could explain problems met during the covid pandemic crisis. This paper proposes to realize the healthcare digital transformation for increasing the hospital's global performance and the level of safety by defining a new methodology in which sustainability (social societal ad environmental aspects) is used as the kernel of the digital transformation process. Indeed, the use of technologies such as the Internet of Things (IoT), Artificial Intelligence (AI), and Big Data just in specific processes makes hospitals unable to effectively implement Healthcare 4.0. The problem lies in the fact that a complete implementation of Healthcare 4.0 beyond digital transformation, implies the integration of technical and sociocultural aspects inside the hospitals. The main barriers are associated with the development of an incorporated IT infrastructure aligned with the hospital's strategy. This issue is proven by the fact that healthcare has been the slowest sector to adopt information technologies.

This paper is based on a research-action project involving the elaboration of a Healthcare logistics 4.0 framework that aims to introduce new concepts and tools in hospital logistics to mitigate the mentioned barriers and allowing the 
implementation of suggested solutions in hospitals for measuring their impact on the healthcare digital transformation. The focus is on improving healthcare performance mainly because human lives depend on having all resources available, at the right time, in the whole hospital.

This new framework will contribute to the better management of hospital processes optimization. These improvements are required due to the aging of the population worldwide (mainly in Europe) and recently, the high demand for hospital resources because of the coronavirus pandemic. The integration of logistics processes applying Industry 4.0 main technologies to improve the efficiency of logistics flows would result in massive gains for hospitals, affecting process efficiency and resulting in cost reduction and ensuring better patient management. Despite the imminent benefits of adopting the mentioned technologies fully in hospital flows, it is not yet clear and easy to understand the real results of implementing technology and how its implementation will affect the flow.

The aim of this paper is to present an intelligent problem-solving tool that will facilitate the identification of logistics deficiencies in hospital flows and propose solutions implementing the lean manufacturing approach and Industry 4.0 main technologies. As a result, a hospital logistics digital twin will be elaborated and will facilitate the test and simulation of scenarios within hospitals, a clear identification of flow deficiencies, and how to solve them based on expert system knowledge.

After a literature review on logistics flow optimization methods, and logistics 4.0 formalisms such as Artificial intelligence (machine learning, expert systems, multi-agent systems), IoTs, and Big data analytics, a sustainable healthcare logistics 4.0 framework will be presented, and the structure of the intelligent software tool being developed for supporting sustainably the healthcare digital transformation will be exposed. The development of the software tool will exploit two hospitals (of the "Grand Paris Sud region") real data acquired during the study. The analysis of hospital flow deficiencies will be supported by the evidencing of real data collected in a hospital. The data collected will be transformed for elaborating a reference model called the "ideal situation" that could be suggested by the software tool during a hospital flow digital transformation. Then, based on the two hospital situations, an illustration as the suggested solution that would be proposed by the tool will be presented.

\section{Literature Review}

Many methods exist for improving company logistics performance such as lean manufacturing and DMAIC (Define, Measure, Analyze, Improve, Control and Standardize) formalisms. They have been exploited to improve hospital flow performance but don't necessarily include the use of industry 4.0 concepts and problems of patient and personal health security are not solved. Indeed, various healthcare experiences are made. For instance, in [1] four digital technologies (real-time care plan development and remote consultations, digital non-invasive 
care, interconnected medical emergency support, and patient data collaborative digital platforms) are indicated for measuring the impact of new technologies on hospital resilience. Basic architecture of healthcare 4.0 [2], information and communication technologies pillars for healthcare 4.0 [3], or three-layer fogcomputing based eHealth architecture [4], have been presented in the literature but include only technological aspects and no logistics expectations.

This section presents some of the concepts, methods, and tools of the literature that could be used for solving the hospital flows problems in the context of Covid pandemic constraints.

\subsection{Logistics Flow Optimization Methods}

Many tools and methods are exploited by industrial companies and contribute to optimize their performance. Most of them include a part of lean manufacturing method such as "Just In Time" (JIT) philosophy (Kanban, SMED), 5 S, Value Stream Mapping (VSM) tool, Waste management (Mudas), etc. Another tool is adapted for logistic problems' solving: DMAIC method. This part presents both methods.

\subsubsection{Lean Manufacturing}

This method is used for identifying added values and non-added values (waste) in a flow and elaborated transformations required for suppressing the non-added values and increasing the added values performance. this methodology is issued from the Toyota Work Philosophy, and the term "Lean" first appeared in the 90s. Seven types of waste (Mudas) were developed: overproduction, wait, transport, stock, unnecessary activity, defects, and motion. Overproduction is the task that generates the most losses [5]. To implement the Lean philosophy, several tools, and techniques such as VSM that helps to distinguish the non-waste and waste processes. The implementation of the Lean Manufacturing approach is realized in three main stages: pre-implementation stage (lean readiness), implementation stage (lean approach), and post-implementation stage (results) [6].

Aiming at increasing process efficiency and mitigating non-added value processes, Lean Manufacturing methods have been extensively implemented in healthcare institutions. For instance, the method is used for collecting patient information several times, unnecessary patient displacement, excessive waiting by patients for appointments, and uncoordinated processes. Despite the wide application of lean assumptions in hospitals and clinics, the prospected results are not achieved [6]. The significant number of failures can be explained by 3 main factors:

- Absence of adaptation: it is essential to adapt lean concepts to knowledgeintensive service sectors such as healthcare. This transition is not always clear, which causes a higher probability of failure.

- Lack of readiness: the unawareness about the Lean Manufacturing approach by most of the employees added to the inefficient training systems are the main bottlenecks encountered for an efficient lean implementation. Main 
problems in lean implementation projects are caused by the lack of corporate culture and change management.

- Lack of systemic approach: the Lean approach is widely used for specific problems, without taking into consideration all the processes.

The use of this method is not sufficient for solving all problems related to covid pandemic context and logistics flows optimization. The following section will focus on the second flow optimization method.

\subsubsection{DMAIC}

Six Sigma is a method used for reducing defect rates, which is based on statistical and scientific methods. The improvement procedure is widely used to increase quality standards by reducing variation and improving complex problems in manufacturing and business firms [7] [8]. Six Sigma defines steps to improve quality and minimize variability in the processes. The four main steps of the method are [7]:

- Function model and objective of the method;

- The DMAIC (Define, Measure, Analyze, Improve, and Control) separating the model in stages;

- Techniques to be employed;

- CTQ (critical-to-quality) characteristics and the difference between the causes (vital and trivial).

A key element of Six Sigma is the DMAIC approach, which consists of understanding root causes of a problem through exhaustive analyses to propose assertive solutions [7] [8]. Its main objective is to apply the five steps (Define, Measure, Analyze, Improve, and Control) structuring a data-based analysis and prioritizing an exhaustive analysis of premature decision making to improve the process.

In the Define phase, the whole project is analyzed from the KPIs to project constraints and risks. The Measure phase determines the process capability and performance through the evaluation of waste, defects, and variations of the project. The Analyze phase seeks to determine the cause-and-effect relationships between steps and results of the process. The root causes for variability, imbalance and waste are identified. In the Improve phase, changes and improvements are elaborated in order to eliminate the root causes of a process. Improvements and changes are tested and implemented [8].

This method is structured and introduces the formalization of the measurement. Key performance can be elaborated in this frame and the structuration of the data associated with each phase could be exploited. But this method is not able to solve all problems related to covid pandemic context and logistics optimization.

The combination of both methods will allow to define an adapted methodology for managing the hospital flows digital transformation.

Indeed, the integration of Logistics 4.0 concepts and new technologies will introduce the digital aspects in the transformation and facilitate the global healthcare logistics performance. 


\subsection{New Technologies and Methods for Logistics 4.0}

\subsubsection{Logistics 4.0 Concepts}

Many frameworks have been developed on industry 4.0 and logistics 4.0 concepts, as explained in [9], but are only focused on technological and organizational parameters or social and human aspects. Then, they are not sufficiently used by SMEs. A framework with sustainability as the kernel of changes that combines both parameters and aspects cited above, has been elaborated. Sustainability as defined in the Cambridge dictionary is the quality of causing little or no damage to the environment and therefore able to continue for a long time. This includes social, societal and environmental dimensions [10]. Instead of focusing on new technologies and organization (like in other frameworks), sustainability is used for taking into account SME brakes for this implementation, defining levers for accelerating dynamics of implementation, and implementing the solution in coherence with the company capacity.

This general approach of industry 4.0 concepts implementation in SMEs is based on the use of GRAI Methodology [11] combined with DMAIC method (Define, Measure, Analyze, Innovate, Control and Standardize), lean Manufacturing methodology (Added and non-added values optimization) and Design of Experiment approach (observation, elaboration, experimentation, and transformation phases).

GRAI methodology developed at the University of Bordeaux has been chosen because it is one of the three main methodologies with PERA [12] and CIMOSA [13] of enterprise modelling. Indeed, CIMOSA (Computer Integrated Manufacturing Open System Architecture) is a methodology used for designing CIM open system architecture, for defining a set of concepts and rules for facilitating the realization of future manufacturing systems, and for aiding the reimplementation of existing systems. CIMOSA is the reference architecture for describing enterprises by giving characteristics for modelling production systems on three levels: definition of requirements, design specification, and implementation description [14]. PERA (Purdue Enterprise Reference Architecture) is used for describing in detail the steps in a project life cycle from the requirements to the end of the project. The approach developed identifies in the project, the functional analysis, the functional design, the detailed design, the development, and the implementation. GRAI methodology associates both previous methods' advantages in the company performance analysis and improvement.

This framework could be used for managing the healthcare logistics digital transformation because it is focused on sustainability (social (personal health and patient), societal and environmental aspects) combined with new technologies and tools such as AI, IoTs and big data analytics.

\subsubsection{Artificial Intelligence Concepts and Tools}

Artificial intelligence (AI) is a technology that adapts very well to the new challenges that emerge in the development of the fourth industrial revolution. 
Within artificial intelligence, cognitive systems and the learning of machines play an important role in the realization of the economy and society of both the present and the future [15]. Machine learning, deep learning, and other techniques have many applications in different domains such as healthcare, surgeries, diagnosis, etc. Even with the advertising created by technology companies such as Google, Facebook, and Amazon about Artificial Intelligence and its applications, there are still many learning applications to be explored, particularly within the construction sector in areas such as planning and site management, health and safety, and forecasting construction costs, which have not yet been so well explored [16].

Within the context of the supply chain and logistics, the use of Artificial Intelligence allows for opportunities to streamline operations by leveraging machine learning methods [17]. The use of AI within logistics consists of the wide use of data about each component of the supply chain [15]. One of the most used algorithms within this field is machine learning (ML), which is able to identify patterns in large data sets without knowledge of the underlying system. Through the analysis of planned and executed historical routes, it aims to identify the contribution of specific customers to the deviations of routes and to identify the constrained customers [17].

In recent years, one of the tools that have stood out in the use of Artificial Intelligence is the multi-agent system (MAS), which has attracted great attention due to its wide application in many fields, such as satellite communications, biological systems, mobile robots. This Multi-agent concept could be used by the software for managing the digital twin elaboration. Indeed, it is integrated with simulation tools (Flexsim, Anylogic, etc.) that could be used.

Based on cloud computing, a predictive control scheme for a multi-agent networked system is proposed to achieve stability and consensus simultaneously, where network delays are actively compensated [18].

At present, Artificial Intelligence has more complex techniques such as artificial neural networks, fuzzy logic, and genetic algorithms, which are common research topics because they can handle complicated problems in prediction and other areas, which are hard to solve by traditional methods. When one compares the capacity of AI techniques with conventional techniques such as ARIMA and Regression in the field of prediction and one notices that systems based on AI have more accurate results in these conventional approaches, which for this reason they have been used successfully in place of the prediction problems [19].

For the definition of an ideal model for healthcare logistics, case-based reasoning and generalization reasoning could be used. They could be combined with digital transformation expert knowledge for realizing the hospital logistics improvements. Indeed, the theory of the expert system could be exploited. The expert system described in Figure 1 is defined as an artificial intelligence system using knowledge from a relatively limited field to solve problems in that field. It 
is used to simulate human experts to solve problems in a professional environment [20]. One of the most important characteristics of an expert system is the ability to make inferences and automated reasoning [21].

To understand expert systems, certain concepts need to be defined, first, data and their relationship with knowledge. If, in the past, processing in a computer consisted of performing operations on data, with man understanding the meaning of manipulations, the objective of designing programs capable of analyzing the semantics (meaning) of the data used implies the use of knowledge bases. Such programs can produce logical chains from the conclusions obtained beforehand, as if passing this knowledge through the conclusions, verifying their logic, specifying, and building finer structures [22].

In many decision-making situations, expert systems have been helpful in deducing a more conceptual understanding from data. With the rise of the Internet of Things and the increasing presence of cloud computing, expert systems need to be improved to support higher-level operators, large rule bases, and ample flow of inputs [23]. These systems include a human-computer interface, knowledge management system and library, interpretation module, inference engine, and dynamic database [20].

Expert systems involve three main knowledge: facts, rules (know-how), and control structures. The knowledge of facts is communicated to specialized systems by an expert geographer during a dialogue and reflects the views of this man during this work. The knowledge of the rules is closely linked to that of the facts but is the result, so to speak, of the accumulation of this knowledge, which is used to elaborate the rules that define the behavior of the system. Finally, the knowledge of control allows the choice of the best strategy for the functioning of the system [24].

An expert system can solve problems by using reasoning from known facts and rules. A rule-based expert system consists of five elements presented in Figure 1:

- The knowledge base: the knowledge base contains the knowledge about the domain;

- The rule base: it is used to compare the model with the theoretical model through rules;

- The inference motor: provides reasoning, so that an expert system can reach a solution.

- The explanation: provides the user with the answer to the question of why the solution is reached.

- The user interface: the user interface allows the user to interact with the other components of the expert systems.

The Expert System must manage inputs from the user interface, to make fuzzification, to use Inference Motor to reach a solution, to make defuzzification, to use adapted knowledge, and to manage outputs in the user interface.

All the formalisms presented above are used in the literature for treating 


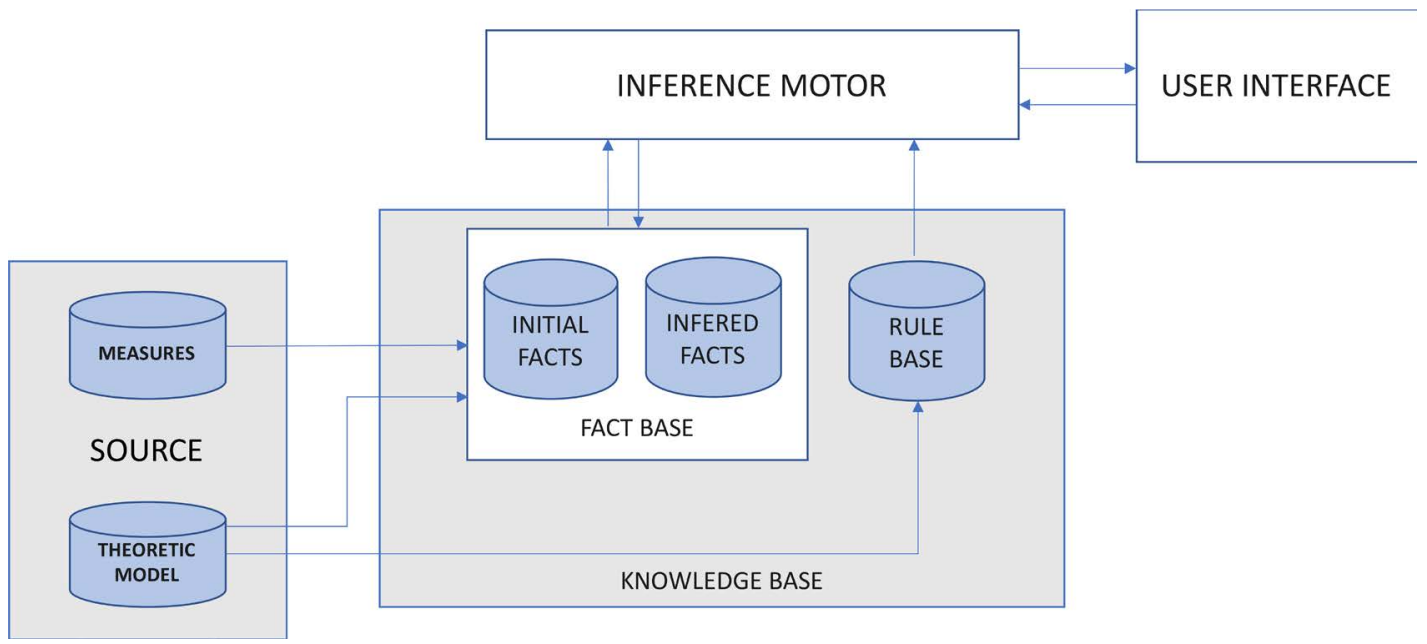

Figure 1. Expert system general architecture.

healthcare problems but need to be combined with other new technologies. Indeed, physical measuring systems must be elaborated.

\subsubsection{Internet of Things}

According to [24] the Internet of Things (IoT) is an organization of correlated preparing devices, mechanical and propelled machines, things, animals, or people that are given unique identifiers and the ability to move data over a framework without anticipating that human-to-human or human-to-PC. The Io $\mathrm{T}$ architecture is designed to provide all objects with identification, detection, networking, and processing capabilities, so that they can exchange and share information with each other and develop advanced services over the Internet. As described in [25], IoT architecture involves four levels:

- hardware: smart devices such as sensors and actuators;

- connectivity and communication middleware (data collected by sensors and stored in the cloud);

- big data storage and analytics (storage and analysis of data; data analytics is the process of transforming data from raw units into actions and insights);

- and IoT applications (various applications leverage across several sectors such as transportation, logistics, delivery, healthcare, etc.).

The elaboration of the IoT physical systems in healthcare 4.0 problems is pertinent and regularly used. But it is important in this case to manage all data associated with these measures.

\subsubsection{Big Data and Analytics}

$\mathrm{BDA}$ is defined as a holistic approach for managing, processing, and analyzing data sizes (volume, variety, velocity, veracity, and value) that are needed to create action-oriented information for sustained delivery, performance measurement, and competitive advantage [26] [27]. Analytic problems and techniques [28] are summarized in Figure 2.

An analytics project will try to create a data model from unstructured data. 


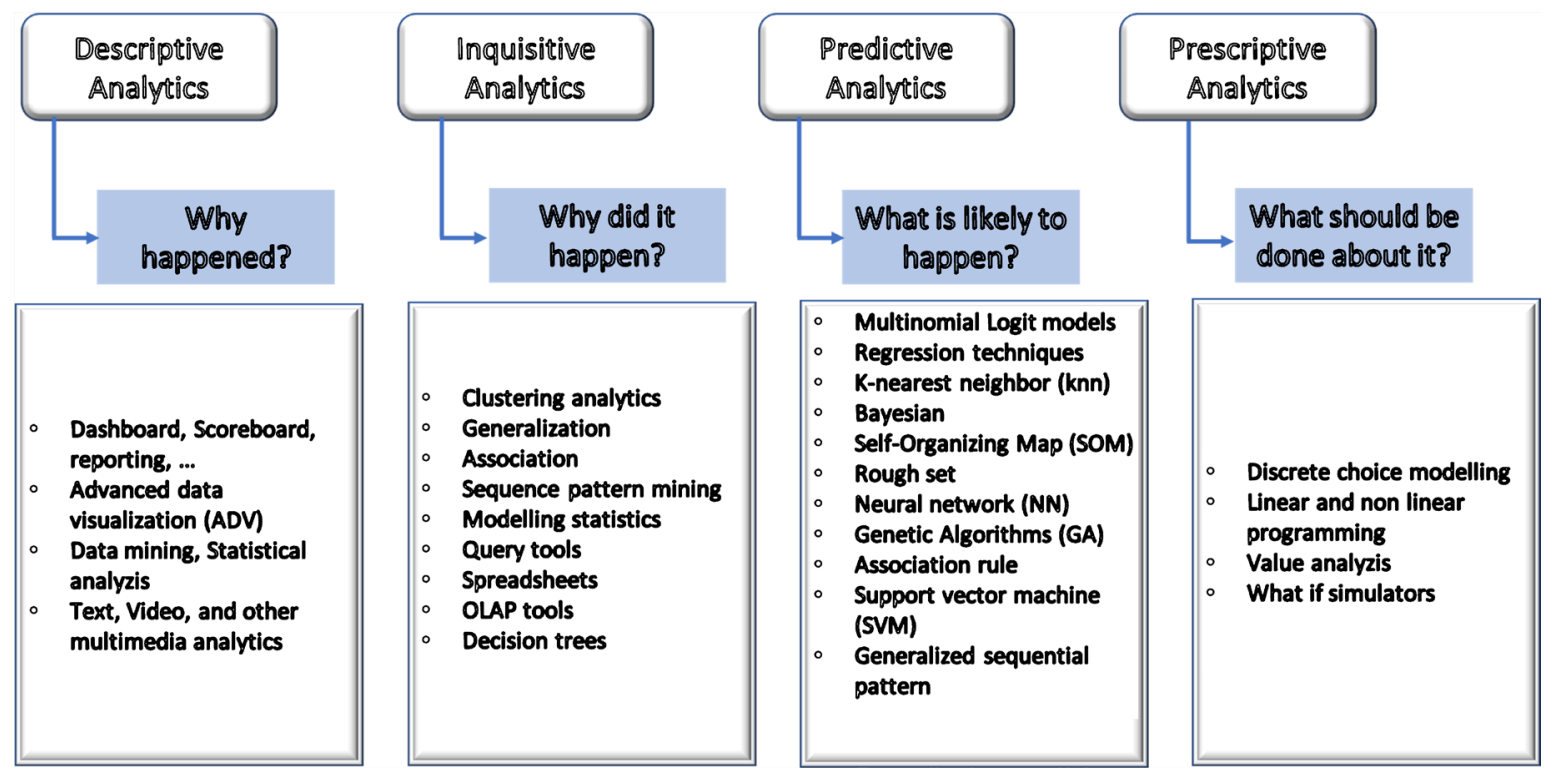

Figure 2. Classification of big data analytic problems and techniques.

Unstructured data is another area in most organizations, but it too can provide important insights and can also help add a qualitative story to quantitative analysis. That means that when combined with intuition, the data becomes a powerful tool for making decisions for business. As such with the perspective to produce services to logistics managers or company logistics analytics uses data to make better decisions. For instance, relating to a company, one of the most critical decisions in transportation and logistics involves planning for the future. Managers need to prepare for a host of what-if scenarios, understanding how each action they take could potentially impact their network and costs. It is overarching to indicate that external consulting services are conceptualized for logistic firms, through software services.

The intelligent support system that is being developed, is based on AI formalisms, more precisely the use of expert systems, machine learning and multi-agent systems to solve healthcare logistics problems. The objective is to demonstrate that some of the concepts presented above could be exploited for creating and developing an efficient intelligent support system.

In synthesis, the software tool developed by combining the concepts presented above will be used for transforming digitally the hospital logistics. The following section presents how the formalisms, methods, concepts, and tools explained above are exploited for developing the framework of healthcare logistics 4.0 and the intelligent software tool for optimizing this hospital logistics flow.

\section{Healthcare Logistics 4.0 Optimization Method and Tools}

The decision-aided system is a problem-solving tool based on Artificial Intelligence designed to improve logistics in hospitals. The aim of the decision aided system is to eliminate inconsistencies in hospital flows and optimize the lead time throughout the simulation and measure of the gains generated by the im- 
plementation of logistics 4.0 main technologies. The hospital transformation by the full implementation of Healthcare 4.0 concepts and methods is represented in the framework of Figure 3.

\subsection{The General Approach of Healthcare 4.0 Optimization}

This tool is developed from the collection and analysis of hospital flow data and the identification of numerous dysfunctions and the opportunity to implement concepts and technologies to reduce non-value added and reduce costs. The development phases of the tool are shown in Figure 4.

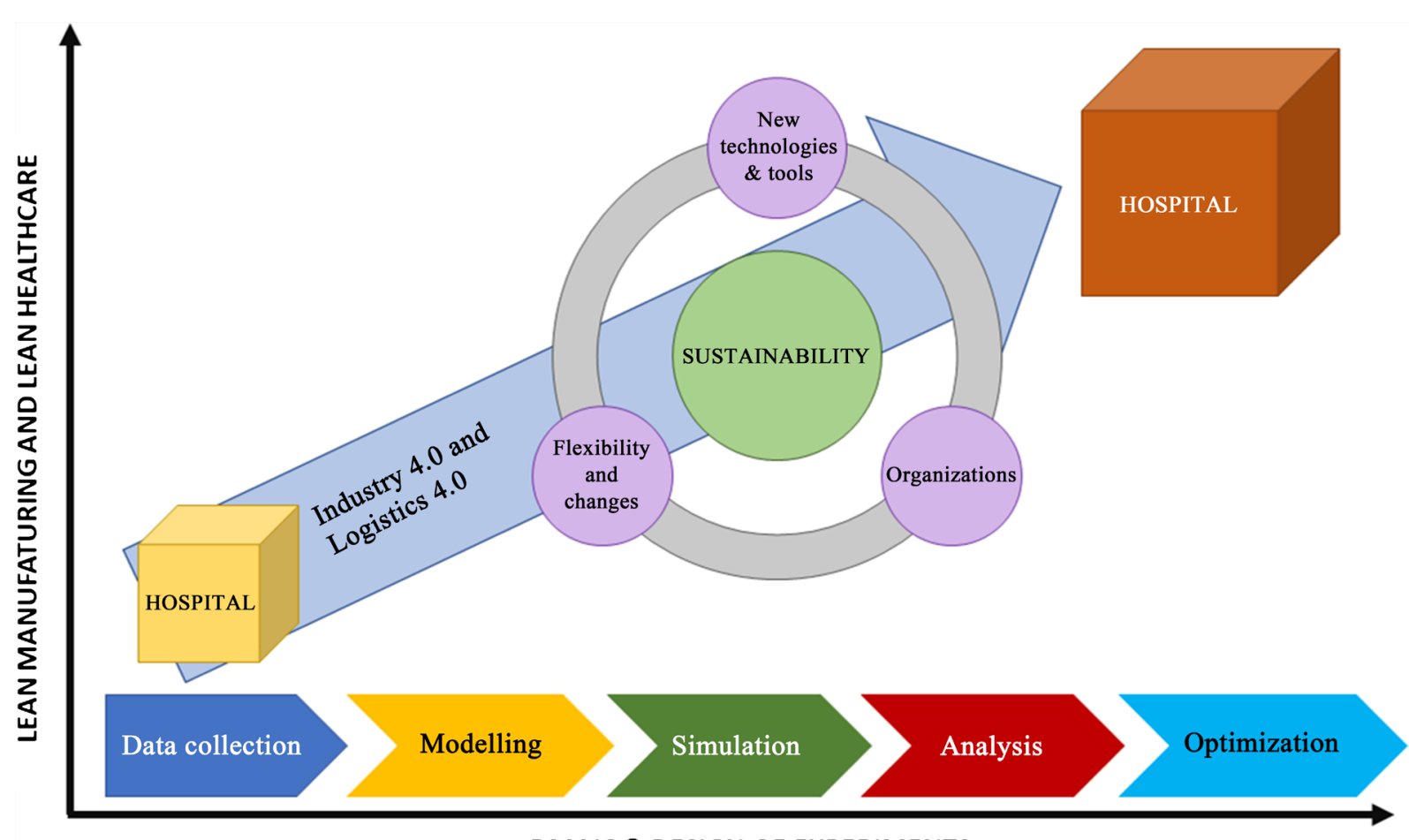

DMAIC \& DESIGN OF EXPERIMENTS

Figure 3. Framework for healthcare 4.0 concepts implementation.

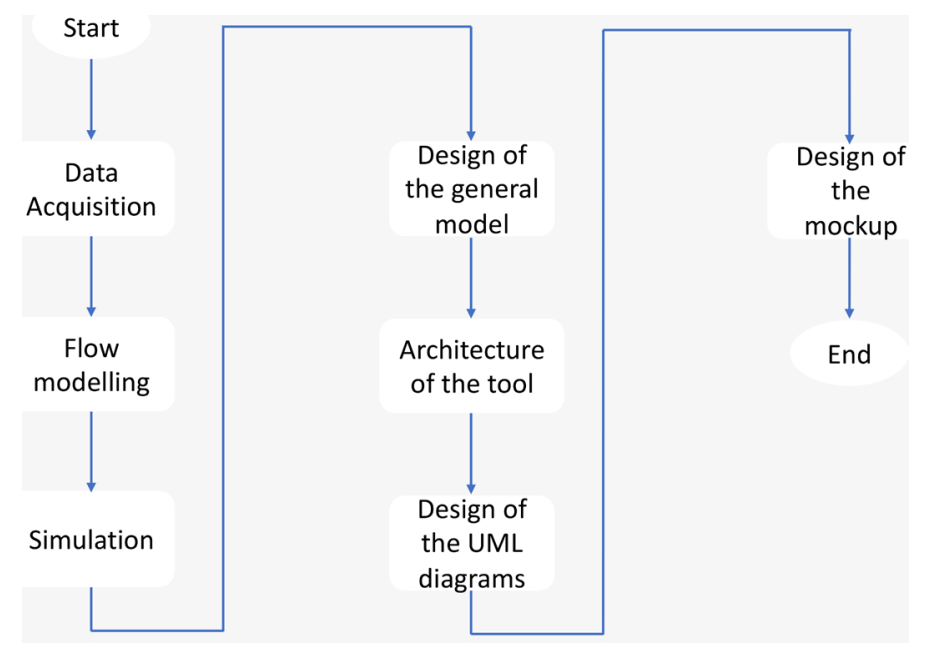

Figure 4. Tool development phases. 
- Data acquisition: The data collected in the hospitals was analyzed to verify the possibility of modeling and simulation;

- Flow modelling: The selected flows were modelled using the BPMN method to fully understand the processes and to facilitate the identification of dysfunctions;

- Simulation: Flows were simulated after being modelized to facilitate the identification of dysfunctions;

- Design of the general model: After identifying the main dysfunctions due to the modeling and simulation, the "ideal situation" was determined by applying lean manufacturing and logistics 4.0 concepts. The general model is being used as an ideal situation in the conception of the tool;

- Architecture of the tool: The structure of the decision aided system being developed supporting the logistics 4.0 and lean manufacturing concepts implementation in hospital flows;

- Definition of the UML diagram: In order to support programmers during software programming each part of the tool was detailed showing the expected inputs and outputs;

- Construction of the maquette of the tool: The visual part of the tool architecture was modeled in order to facilitate the programming and the understanding of the link between the final product and the UML diagram.

To reach its goal, the software tool requires data from hospitals as inputs. These inputs will be compared to the general model (ideal situation) created from the real data collected in hospitals. The outputs of the intelligent system show the main dysfunctions of the processes and give suggestions incorporating logistics 4.0 main technologies.

The inputs of the intelligent system are:

- Layout of the hospital;

- Place of beginning and end of the activity;

- Number and type of healthcare workers;

- Type of process;

- Times per day and week that the process is performed;

- Equipment used in the process;

- Duration of the process.

The outputs of the intelligent tool are:

- Dashboard with main KPIs for flow analysis in the current process;

- Simulation of the current process;

- Solutions according to 4.0 logistics parameters (e.g. use of AGVs in the flow for transportation instead of people);

- Benefits (in terms of defined KPIs to reduce non-added value time) of adopting each one of the solutions proposed;

- Dashboard for flow analysis after the implementation of the tools chosen by the user;

- Simulation of the process with the solutions mentioned (use of the digital twin). 
One of the main purposes of the tool is to present solutions and be flexible according to users' choices. The user will be able to simulate and understand the main implications in the process of adopting a solution provided by the software. Therefore, the tool intends to facilitate the implementation of Healthcare 4.0 by transforming the way that the user can reach the gains of introducing its technologies and concepts in the hospitals.

\subsection{Concepts and Methodology for the Intelligent System Devel- opment}

The methods, concepts and tools used for developing Healthcare Logistics 4.0 are elaborated from lean manufacturing concepts, decision aided mechanisms, Industry 4.0 and Logistics 4.0 formalisms. Despite the great application of these concepts and formalisms in industries, the concepts can be adapted to be used in the healthcare sector.

Lean manufacturing concepts were used to minimize non-added-values, waste and focus on the improvement of value. The greatest needs for improvement in the flows of the hospitals analyzed are:

- Unnecessary transport;

- Manual tasks that could be digitized;

- Lack of organization of the process;

- Lack of traceability of medicaments and other supplies;

- Unnecessary displacements.

Logistics 4.0 implementation in this scenario would result in the elimination of all non-added values and optimization of all processes. For example, to avoid food transportation, which is a non-added value movement, it is possible to assign this task to an AVG. This change would optimize the process and the professionals responsible for this process could use their time for tasks that have the patient as the main priority.

The framework being developed in the project combines the technical aspects related to the use of Healthcare 4.0 formalisms in the processes for increasing performance and the sustainability aspect with patients and hospital workers at the center of the transformation. The efficiency of healthcare services must be improved to guarantee the quality of patients' treatments and the workers' well-being.

The intelligent support system is being developed using Artificial Intelligence formalisms to integrate human reasoning into the system to improve its efficiency and support the suggestions given. The system needs to be capable of reasoning in order to solve problems, acquiring new knowledge and applying it into a system, exploiting real cases to learn and understand how to solve different situations, measuring the importance degree of the situation and treating incomplete/wrong data with high efficiency.

\subsection{The Intelligent Support System for Healthcare Logistics 4.0}

The structure presented in Figure 5 supports the development of the decision 


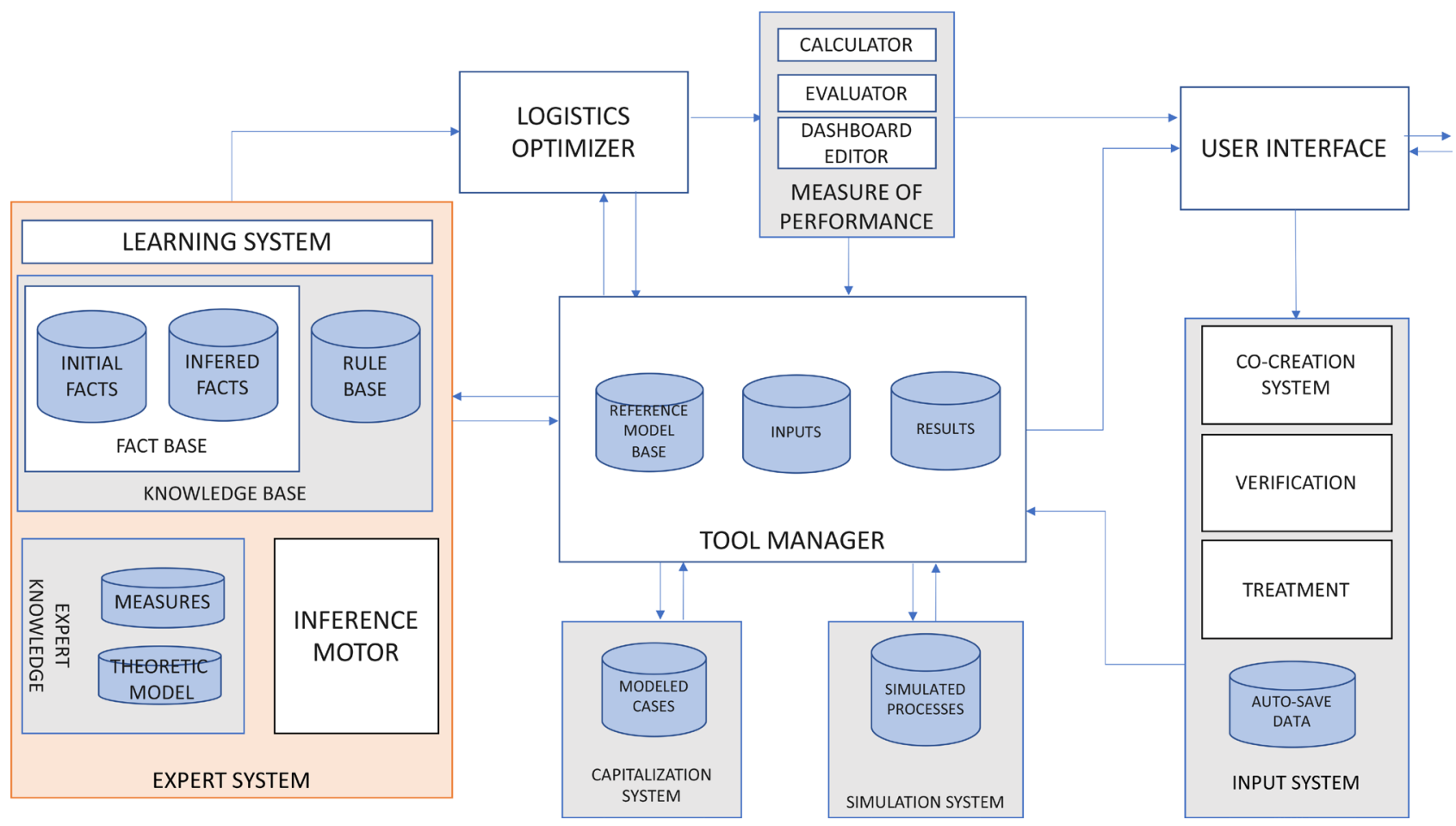

Figure 5. Architecture of the intelligent support tool.

aided tool. The proposed architecture is elaborated for structuring data collected in hospitals and transforming them to support the implementation of Healthcare 4.0. The modules are linked to facilitate the demonstration of the data flow in the software.

\subsubsection{Database}

The first step to find a solution for the hospital's logistics problems is to analyze the actual situation comparing it with an ideal situation. The Database will store the ideal situation in the Reference Model and the actual situation will be recorded in the Inputs of the system. The software will be able to cross-reference these data to clearly define the problem and then propose solutions. The outputs of the comparative analysis will be recorded in the Results. The database will be the fixed memory of the whole platform, which means that some modules will have access to this data to function properly.

\subsubsection{Tool Manager}

This module manages the interactions between the different modules of the platform. It serves as a link between them and could be used to make the final synthesis. It is essential for the functioning of the platform since it serves as a link between the other modules.

\subsubsection{The User Interface}

The user interface manages the interaction between the user and the platform. It is through this module that users can enter their data, understand the problems identified in the current situation, participate in the creation of the problem res- 
olution through the choice of options and see the results after their implementation.

\subsubsection{The Expert System}

The expert system of the intelligent tool must be able to acquire new knowledge depending on the situation detailed by the user. The user will contribute to the improvement of this module due to the flow data that will be inputted in the software. The software will accumulate and sort the data, being able to propose more relevant solutions as it is used.

The inference motor is the kernel of the expert system. The interaction between fact and rules bases, are created by this module of the Expert System. Data stocked in these bases would be extracted for analyzing the current case inputted by the user and identifying problems. Basically, it is a protocol for navigating inside the database through rules aiming to solve the problems.

The aim of the Learning System is to improve Expert System performance through the Artificial Neural Network. The module will be able to forecast the demand and adapt the formulas by learning with data provided by the user. Data available integrated with the tool in the Inputs would increase the learning system knowledge and improve its performance.

\subsubsection{The Logistics Optimizer}

The Logistics Optimizer module is directly linked to the following modules: measure of performance, the database, and the expert system. Its main purpose is to solve problems detected in the expert system module. The outputs of the module are the improvements that will be proposed to the user. The system will then, after the analysis by the expert system, propose solutions. It will also propose a base of possible solutions allowing to choose the appropriate ones according to the perception of the user. To better accomplish these goals, it will also exploit the reference models of the Capitalization System.

\subsubsection{Measure of Performance}

The main goal of the module is to exploit the results of the Logistics Optimizer. This module aims to measure the level of each indicator that has been defined to analyze the flow, this will allow to easily identify the impact of the outputs of the Logistic Optimizer (options of improvement). The outputs of this module must be the indicators related to the process (current and with the improvements chosen by the user) organized in an easy-to-view dashboard.

\subsubsection{Capitalization System}

This module will be used as a database of old cases that can be accessed by the user to compare them with the current situation of the logistic flow of the hospital. These cases can give examples of how implementation of 4.0 logistics technologies can improve real processes.

\subsubsection{Simulation System}

The Simulation System module explains the different functions that need to be developed to properly connect the tool with the source software that will be used 
to simulate logistic flows.

\subsubsection{Input System}

To allow the construction of the final solution to the problems identified by the Expert System together with the user, the module will give the system flexibility of option choice. The module is composed of three parts: co-creation system, verification, and data treatment. The co-creation system will guide the user through various instructions to show him the possible application flow improvement options.

\section{Design of the Intelligent Support System}

To help the software developers for specifying, visualizing, constructing, and documenting the artifacts of the software system the Unified Modeling Language (UML) started to be defined by specifying the main functions of the modules. In the construction of the UML, the main functions of each module were specified and then the secondary functions were detailed in other modules linked to the primary functions. The main functions of each module are representing in following Tables 1-4.

Table 1. Description of the tool manager function.

\begin{tabular}{cr}
\hline Main Function & Tool manager \\
\hline 1 & Link between each module for the proper functioning of the software \\
2 & Enable the system to make the final synthesis \\
\hline
\end{tabular}

Table 2. Description of the user interface function.

\begin{tabular}{cl}
\hline Main Function & \multicolumn{1}{c}{ Module: The user interface } \\
\hline 1 & $\begin{array}{l}\text { Enter input data: allows users to enter their data to login; } \\
2\end{array}$ \\
3 & $\begin{array}{l}\text { Enter hospital data: allows users to enter data on the current status of hospital } \\
\text { logistics }\end{array}$ \\
4 & Show the possibilities of improvement that the user can choose from \\
5 & $\begin{array}{l}\text { Visualization of simulations: Visualize the simulation of the current process; } \\
\text { chosen }\end{array}$ \\
6 & $\begin{array}{l}\text { Show the dashboard with key parameters for each process: these indicators will be } \\
\text { shown after the simulation of the current process and after the implementation of } \\
\text { the improvements, to allow the analysis of the process evolution }\end{array}$ \\
\hline
\end{tabular}

Table 3. Description of the expert system function

\begin{tabular}{cl}
\hline Main Function & \multicolumn{1}{c}{ Expert System } \\
\hline 1 & $\begin{array}{l}\text { Process and analyze inputs: this module will analyze and process the data to } \\
\text { provide the best possible solution }\end{array}$ \\
2 & $\begin{array}{l}\text { Improve the ideal model: through repeated situation analysis, the tool will be } \\
\text { able to propose an increasingly performing solution }\end{array}$ \\
\hline
\end{tabular}


Table 4. Description of the logistics optimizer function.

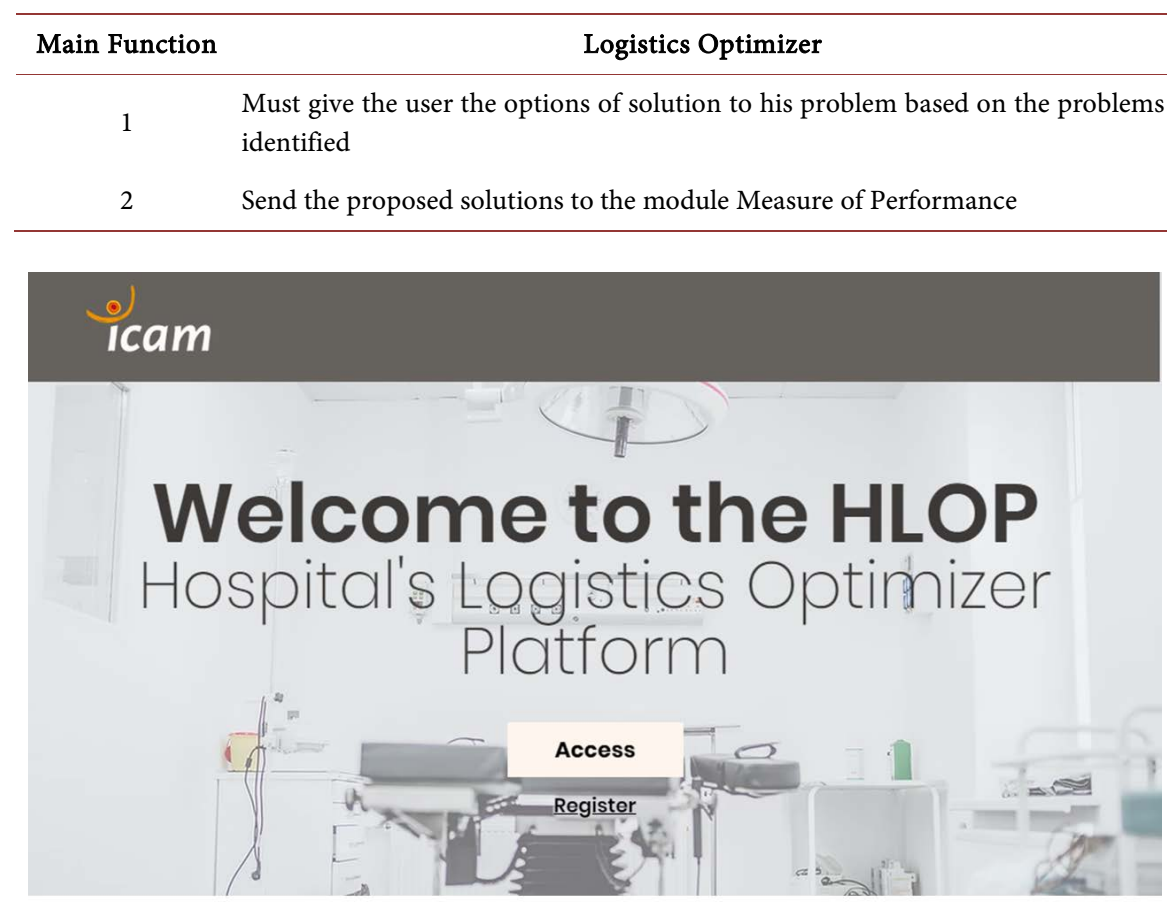

Figure 6. Homepage of the platform.

The maquette of the software was developed to support the visualization of each module and how the integration between them is accomplished (Figure 6). The UML combined with the maquette will allow the development of the software.

\section{Developing the Intelligent Support System}

The last part of this paper concerns the development of the software tool. A hospital digital transformation could be done by simulating the existing system, analyzing this system, and exploiting the ideal situation developed in the software tool for suggesting transformation solution. Two hospitals of the "Grand Paris Sud" region have been studied in detail for elaborating their existing logistics systems. These data have been exploited for finding inconsistencies and points to improve. Both existing systems have been used for defining the digital transformation general model. The actual digital transformation general model will be improved by integrating other cases in the system according to the software tool learning system. The use of the general framework allows to obtain digital transformation steps for optimizing these processes. Each process is studied in detail and transformed progressively with sustainability as the kernel of the transformation. Similar cases of transformations will be proposed to the user for defining the best and adapted organization. The software tool steps contribute to validate the two hospitals' global transformations. And these new transformations were stored as transformation cases for future studies.

The process between entering the information in the software and obtaining 
the final result will be detailed, being possible to identify the different modules of the system through what will be shown in the user interface.

The first step is the identification of the user. The data entered will be stored in the Inputs base (Database module). The objective is that all elaborated transformations will be recorded and able to be reused as a case (Case-based reasoning) for future transformations. In the second page, the user can choose between "create a new process" and "my personal area". In the personal area, as shown in Figure 7, the old, simulated flows will be stored (Results Database).

To create a new process and simulate the current flow to identify its dysfunctions, the user can press "create a new process" (Figure 8). To precisely measure the flow characteristics, the user must enter information as: Type of process (it will be linked to the Simulation System module to identify the types of existing process in the simulation software), Times per day (daily periodicity of the process), Times per week (weekly periodicity of the process) and the layout of the hospital. The information is important for measuring the existing system

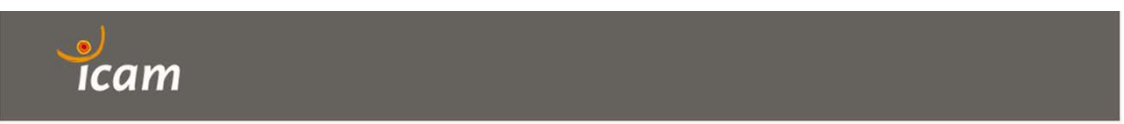

\section{Personal space} Creat a new processus

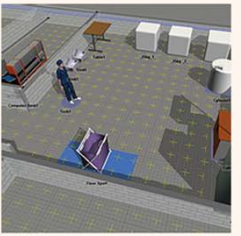

Blanchisserie

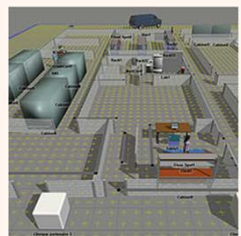

Anticancereux

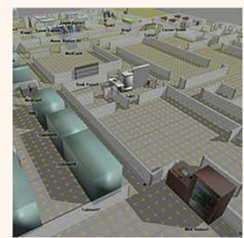

DMS

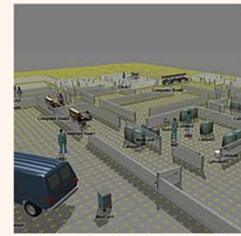

Solutes

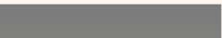

Figure 7. Personal space in the software.

\section{๑)}

\section{Icam}

Myspace

Figure 8. Creating a new process in the software. 
performance and comparing them to the potential transformation results. Each process will be elaborated, measured, and compared with the future solution.

Each flow must be separated into steps. So, the software will be able to simulate the process precisely and, through the module Expert System, identify the dysfunctions. Through the module Logistic Optimizer, it will be able to solve them. The steps require information details about the flow, such as the Duration of the Action, the Equipment used in the process and the Number of workers. Indeed, in the software tool these data have been defined for the ideal solution and the existing information of the new case will be compared to them. It is also necessary to indicate the starting point of the step and ending location as indicated in Figure 9.

The first output of the system will be the simulation of the current process and the dysfunctions found in the flow (Figure 10) according to rules saved in the expert system. The module Expert System will help the identification of the

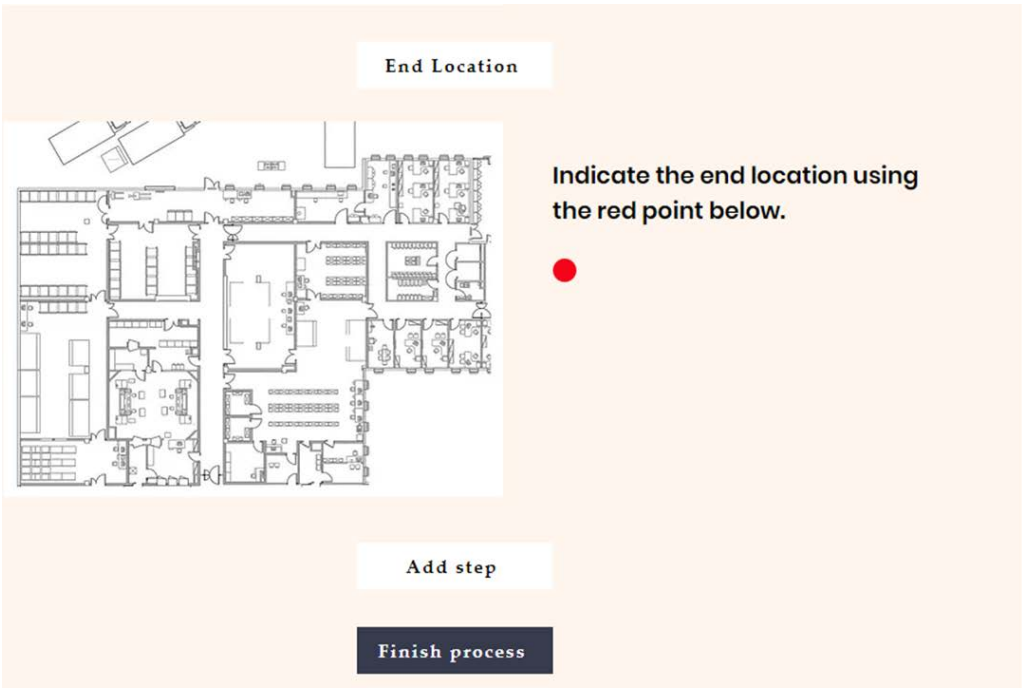

Figure 9. Indicate the ending location in the hospital flow.

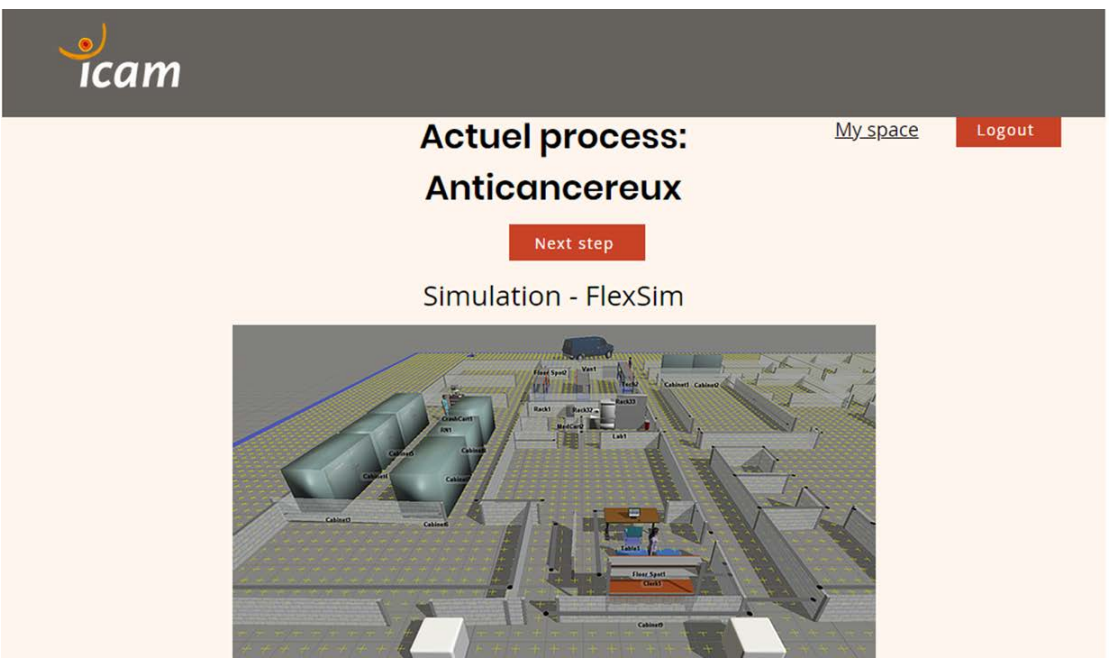

Figure 10. Current process analysis. 
dysfunctions, the Measure of Performance module will present the results of the analyzed flow in a dashboard to facilitate further comparison, the module Simulation System will support the interaction between the simulation software and the decision aided tool.

Thus, the user doesn't need to be a specialist in simulation before realizing the study. A graphical editor will be given to the user and the decision aided tool will help him with suggestions.

The second output is the results of the Logistics Optimizer analysis. The module will propose several solutions that can be implemented to improve the flow performance and the solutions must include the adoption of Logistics 4.0 main technologies. The Input System module will allow the co-creation of the solution with the user. Among the proposed solutions, the user can decide which are the most applicable depending on their context. For instance, in the situation presented in Figure 11, the user can decide between adopting the AGV for locomotion, optimization of locomotion and reorganization of materials. All the improvements have an impact on the flow that must be shown to the user.

The flow will be simulated again with the improvements chosen by the user. The user will have the flexibility to simulate and test different scenarios from the options he chooses. The simulation (Figure 12) and the dashboard (Figure 13)

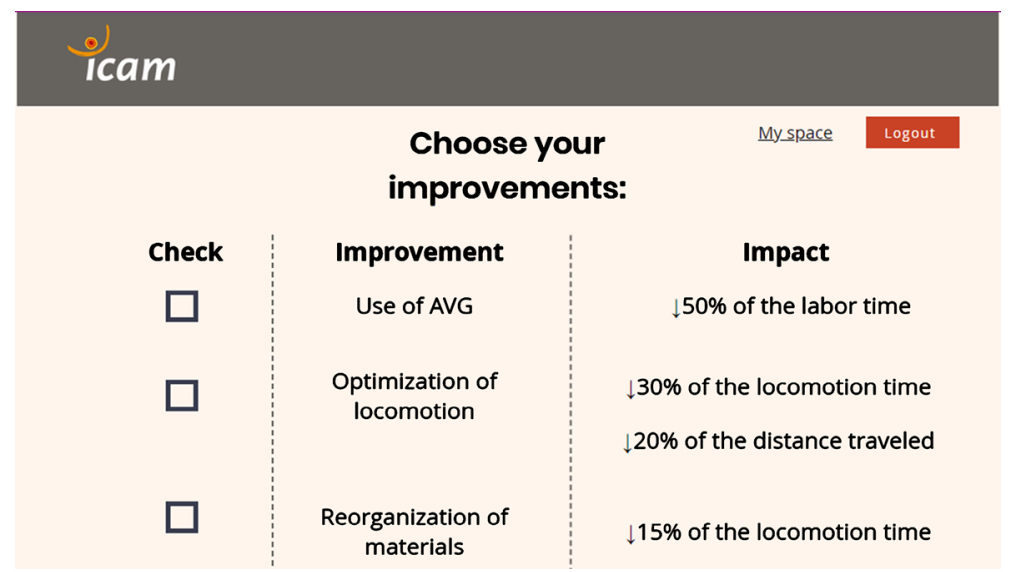

Figure 11. Choice of applicable flow improvements.

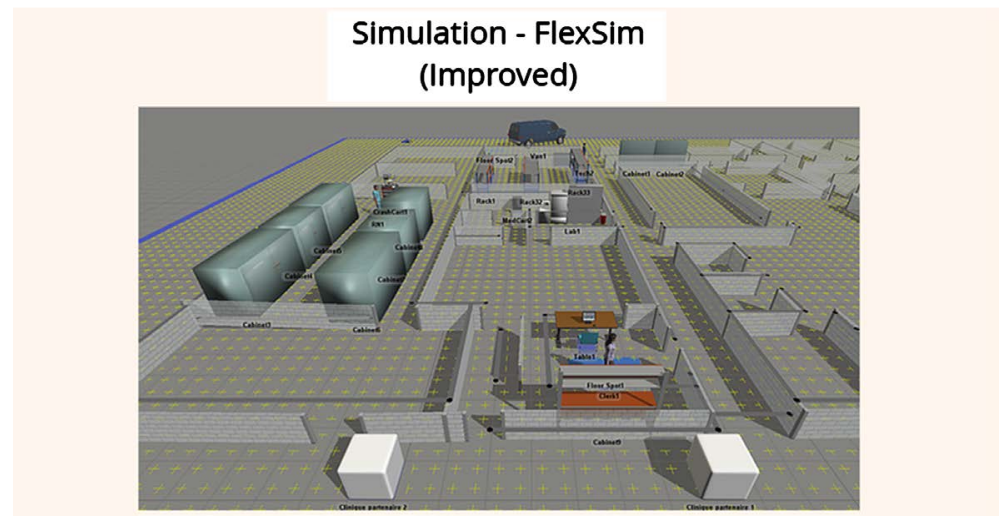

Figure 12. Final output (simulation) of the decision aided tool. 


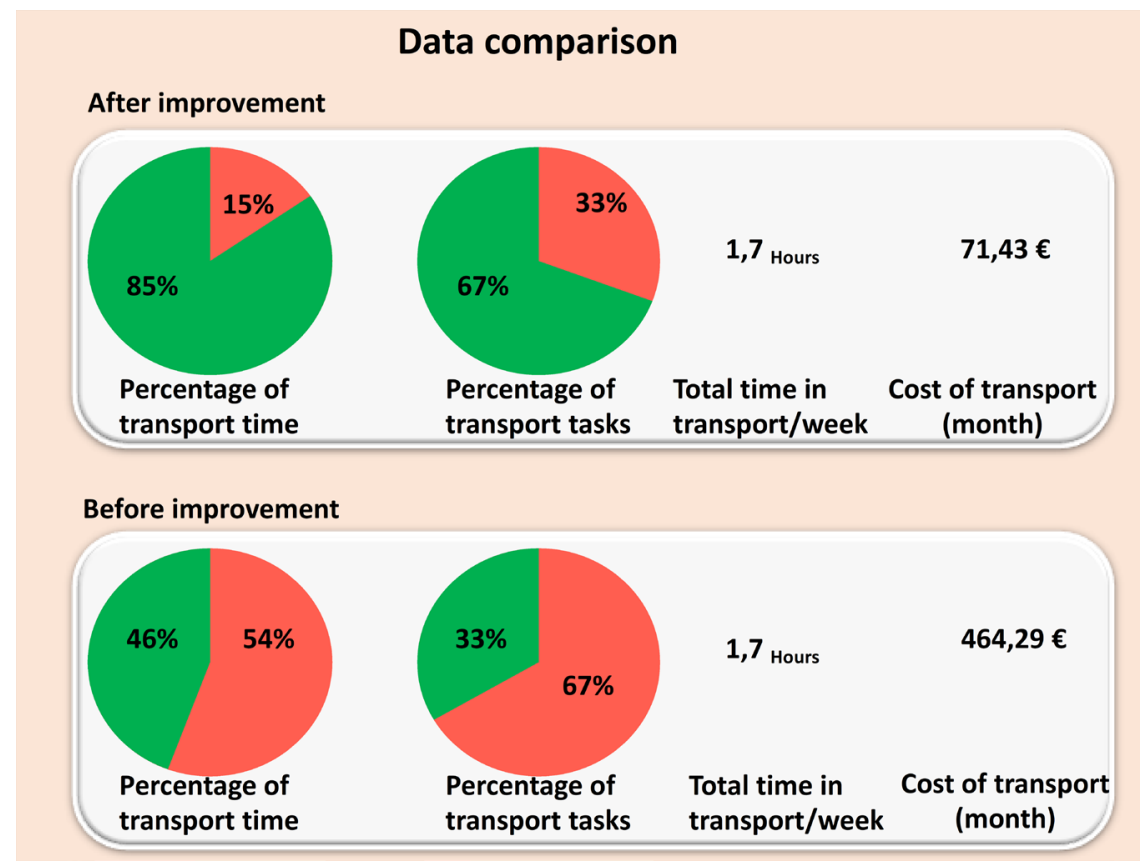

Figure 13. Final output (data) of the decision aided tool.

comparing the current situation and the improved process are outputs of the Simulation System and Measure of Performance modules respectively.

In the end of the user experience, it is possible to evaluate the software and save the results. The simulated flow will be recorded in the Capitalization System to be accessible to other users who will simulate other flows. Then, the general healthcare logistics 4.0 framework contributes to define the new digital transformation, with a co-creation process between the software tool and the user. Added values and non-added values of each process, are determined by the tool in comparison with dereference model. The collaboration with the tool during the transformation is complete. The user knowledge and the new transformation will be integrated and will increase the reference model defined in the software.

This general description of the intelligent support tool utilization for optimizing healthcare logistics 4.0, contributes to the real development of the software. This development is ongoing.

\section{Conclusions}

Considering the great importance of the integration of all flow data in a hospital to the hospital logistics performance, and additional constraints integrated in hospitals by covid pandemic, this paper aims to present an intelligent software tool that makes the identification of dysfunctions in the hospitals flows easier and the adoption of the Industry 4.0 technologies to solve these problems more tangible to users. The healthcare logistics digital transformation will contribute to reduce nurses, caregivers, and doctors' wasting time, and to increase patient service quality. The imminent need to develop a tool such as the one presented in this paper is based on the analysis of real hospital flows, which presented nu- 
merous dysfunctions.

The flows studied allowed the construction of a general model (ideal organization) that can be used as a support for the hospital's digital transformation. The general model comprises Industry 4.0 concepts combined with lean manufacturing concepts for the improvement of hospital flows. The aim of this article is to show the possibility of automating the implementation of Healthcare logistics 4.0 in a simple and flexible way for the hospital.

\section{Conflicts of Interest}

The authors declare no conflicts of interest regarding the publication of this paper.

\section{References}

[1] Tortorella, G.L., Saurin, T.A., Fogliatto, F.S., Rosa, V.M., Tonetto, L.M. and Magrabi, F. (2021) Impacts of Healthcare 4.0 Digital Technologies on the Resilience of Hospitals. Technological Forecasting and Social Change, 166, Article ID: 120666. https://doi.org/10.1016/j.techfore.2021.120666

[2] Hathaliya, J.J. and Tanwar, S. (2020) An Exhaustive Survey on Security and Privacy Issues in Healthcare 4.0. Computer Communications, 153, 311-335. https://doi.org/10.1016/j.comcom.2020.02.018

[3] Aceto, G., Persico, V. and Pescapé, A. (2020) Industry 4.0 and Health: Internet of things, Big Data, and Cloud Computing for Healthcare 4.0. Journal of Industrial Information Integration, 18, Article ID: 100129. https://doi.org/10.1016/j.jii.2020.100129

[4] Kumari, A., Tanwar, S., Tyagi, S. and Kumar, N. (2018) Fog Computing for Healthcare 4.0 Environment: Opportunities and Challenges. Computers \& Electrical Engineering, 72, 1-13. https://doi.org/10.1016/j.compeleceng.2018.08.015

[5] Cortes, H., Daaboul, J., Le Duigou, J. and Eynard, B. (2016) Strategic Lean Management: Integration of operational Performance Indicators for strategic Lean management. IFAC-PapersOnLine, 49, 65-70. https://doi.org/10.1016/j.ifacol.2016.07.551

[6] Ohno, T. (1988) Toyota Production System: Beyond Large Scale Production. Productivity Press, Portland.

[7] de Mast, J. and Lokkerbol, J. (2012) An Analysis of the Six Sigma DMAIC Method from the Perspective of Problem Solving. International Journal of Production Economics, 139, 604-614. https://doi.org/10.1016/j.ijpe.2012.05.035

[8] Leaphart, C.L., Gonwa, T.A., Mai, M.L., Prendergast, M.B., Wadei, H.M., Wadei, H.M., et al. (2012) Formal Quality Improvement Curriculum and DMAIC Method Results in Interdisciplinary Collaboration and Process Improvement in Renal Transplant Patients. Journal of Surgical Research, 177, 7-13. https://doi.org/10.1016/j.jss.2012.03.017

[9] Dossou, P.E. (2019) Development of a New Framework for Implementing Industry 4.0 in Companies. Procedia Manufacturing, 38, 573-580. https://doi.org/10.1016/j.promfg.2020.01.072

[10] Dossou, P.E. (2018) Impact of Sustainability on the Supply Chain 4.0 Performance. Procedia Manufacturing, 17, 452-459. https://doi.org/10.1016/j.promfg.2018.10.069

[11] Doumeingts, G., Ducq, Y., Vallespir, B. and Kleinhans, S. (2000) Production Man- 
agement and Enterprise Modeling. Computer in Industry, 42, 245-263. https://doi.org/10.1016/S0166-3615(99)00074-3

[12] Williams, T.J. (1994) The Purdue Enterprise Reference Architecture. Computer in Industry, 24, 141-158. https://doi.org/10.1016/0166-3615(94)90017-5

[13] Jorysz, H.R. and Vernadat, F.B. (1990) CIM-OSA, Part 1: Total Enterprise Modelling and the Function View. International Journal Integrated Manufacturing, 3, 144-156. https://doi.org/10.1080/09511929008944444

[14] Chen, D., Doumeingts, G. and Vernadat, F.B. (2008) Architectures for Enterprise Integration and Interoperability. Past, Present and Future. Computers in Industry, 59, 647-659. https://doi.org/10.1016/j.compind.2007.12.016

[15] Oleśków-Szłapka, J., Wojciechowski, H., Domański, R. and Pawłowski, G. (2019) Logistics 4.0 Maturity Levels Assessed Based on GDM (Grey Decision Model) and Artificial Intelligence in Logistics 4.0-Trends and Future Perspective. Procedia Manufacturing, 39, 1734-1742. https://doi.org/10.1016/j.promfg.2020.01.266

[16] Akinosho, T.D., Oyedele, L.O., Bilal, M., Ajayi, A.O., Davila Delgado, M., Akinade, O.O., et al. (2020) Deep Learning in the Construction Industry: A Review of Present Status and Future Innovations. Journal of Building Engineering, 32, Article ID: 101827. https://doi.org/10.1016/j.jobe.2020.101827

[17] Snoeck, A., Merchán, D. and Winkenbach, M. (2020) Route Learning: A Machine Learning-Based Approach to Infer Constrained Customers in Delivery Routes. Transportation Research Procedia, 46, 229-236.

https://doi.org/10.1016/j.trpro.2020.03.185

[18] Li, L., Wang, X., Xia, Y. and Yang, H. (2019) Predictive Cloud Control for Multiagent Systems with Stochastic Event-Triggered Schedule. ISA Transactions, 94, 7079. https://doi.org/10.1016/j.isatra.2019.04.011

[19] Hadavandi, E., Shavandi, H., Ghanbari, A. and Abbasian-Naghneh, S. (2012) Developing a Hybrid Artificial Intelligence Model for Outpatient Visits Forecasting in Hospitals. Applied Soft Computing Journal, 12, 700-711. https://doi.org/10.1016/j.asoc.2011.09.018

[20] Hu, L.-Q., He, C.-F., Cai, Z.-Q., Wen, L. and Ren, T. (2019) Track Circuit Fault Prediction Method Based on Grey Theory and Expert System. Journal of Visual Communication and Image Representation, 58, 37-45. https://doi.org/10.1016/j.jvcir.2018.10.024

[21] Thaker, S. and Nagori, V. (2018) Analysis of Fuzzification Process in Fuzzy Expert System. Procedia Computer Science, 132, 1308-1316. https://doi.org/10.1016/j.procs.2018.05.047

[22] Shao, J., Liang, C., Liu, Y., Xu, J. and Zhao, S. (2020) Relief Demand Forecasting Based on Intuitionistic Fuzzy Case-Based Reasoning. Socio-Economic Planning Sciences, 74, Article ID: 100932. https://doi.org/10.1016/j.seps.2020.100932

[23] Anuradha, M., Jayasankar, T., Prakash, N.B., Sikkandar, M.Y., Hemalakshmi, R., Bharatiraja, C. and Sagai Francis Britto, A. (2020) IoT Enabled Cancer Prediction System to Enhance the Authentication and Security using Cloud Computing. Microprocessors and Microsystems, 80, Article ID: 103301. https://doi.org/10.1016/j.micpro.2020.103301

[24] Poli, J.-P. and Boudet, L. (2018) A Fuzzy Expert System Architecture for Data and Event Stream Processing. Fuzzy Sets and Systems, 343, 20-34.

https://doi.org/10.1016/j.fss.2017.10.005

[25] Ben Atitallah, S., Driss, M., Boulila, W. and Ben Ghézala, H. (2020) Leveraging Deep Learning and IoT Big Data Analytics to Support the Smart Cities Develop- 
ment: Review and Future Directions. Computer Science Review, 38, Article ID: 100303. https://doi.org/10.1016/j.cosrev.2020.100303

[26] Wamba, S.F., Akter, S., Edwards, A., Chopin, G. and Gnanzou, D. (2015) How 'Big Data' Can Make Big impact: Findings from a Systematic Review and a Longitudinal Case Study. International Journal of Production Economics, 165, 234-246.

https://doi.org/10.1016/j.ijpe.2014.12.031

[27] Emani, C.K., Cullot, N. and Nicolle, C. (2015) Understandable Big Data: A Survey. Computer Science Review, 17, 70-81. https://doi.org/10.1016/j.cosrev.2015.05.002

[28] Belhadi, A., Zkik, K., Cherrafi, A., Yusof, S.M. and El Fezazi, S. (2019) Understanding Big Data Analytics for Manufacturing Processes: Insights from Literature Review and Multiple Case Studies. Computers \& Industrial Engineering, 137, Article ID: 106099. https://doi.org/10.1016/j.cie.2019.106099 